
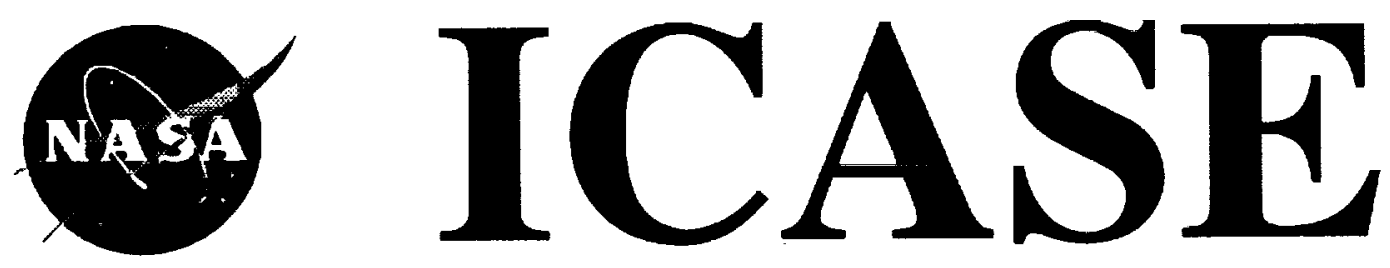

\title{
ALGORITHMS FOR BILEVEL OPTIMIZATION
}

Natalia Alexandrov

J. E. Dennis, Jr. (NASA-CR-194980) ALGORITHMS FCR BILEVEL OPTIMIZATION Final Report (ICASE) $13 \mathrm{p}$

September 1994

Institute for Computer Applications in Science and Engineering NASA Langley Research Center Hampton, VA 23681-0001 



\title{
ALGORITHMS FOR BILEVEL OPTIMIZATION
}

\author{
Natalia Alexandrov \\ ICASE \\ Mail Stop 132C \\ NASA Langley Research Center \\ Hampton, Virginia 23681-0001 \\ natalia@icase.edu \\ J. E. Dennis, Jr. \\ Department of Computational and Applied Mathematics \\ Rice University \\ P. O. Box 1892 \\ Houston, Texas 77251-1892
}

\begin{abstract}
General multilevel nonlinear optimization problems arise in design of complex systems and can be used as a means of regularization for multicriteria optimization problems. Here for clarity in displaying our ideas, we restrict ourselves to general bilevel optimization problems, and we present two solution approaches. Both approaches use a trust-region globalization strategy, and they can be easily extended to handle the general multilevel problem. We make no convexity assumptions, but we do assume that the problem has a nondegenerate feasible set. We consider necessary optimality conditions for the bilevel problem formulations and discuss results that can be extended to obtain multilevel optimization formulations with constraints at each level.
\end{abstract}

Research supported by the National Aeronautic and Space Administration under NASA Contract No. NAS119480 while the first author was in residence at the Institute for Computer Applications in Science and Engineering (ICASE), NASA Langley Research Center, Hainpton, VA 23681-0001.

Second author's research supported by the Slate of Texas under contract \#1059, the Air Force Omice of Scientific Research under F49629-9310212, the Department of Energy under grant DE-FG005-86ER25017, and the National Science Foundation under cooperative agreement CCR-9120008. 



\section{Introduction}

We are interested in nonlinear multilevel optimization (MLO) problems, in general, and bilevel optimization (BLO) problems, in particular, for two related and important reasons. First, general multilevel optimization problems arise in the course of decomposition of multidisciplinary design optimization problems (see, for example, Sobieszczanski-Sobieski, James, and Dovi [15], Sobieszczanski-Sobieski, James, and Riley [16], Sobieszczanski-Sobieski [14], Barthelemy [4], Padula and Young [10]).

The other, related, application is the field of multicriteria (or multiobjective, or vector) optimization. Design of any feature of a complex system involves achieving a compromise among several, possibly competing, objectives. For example, aeronautical design objectives include such criteria as minimizing weight for a given performance, maximizing lift, finding the shape with least drag, achieving the least time trajectory between two points, and other objectives.

There are several current approaches to solving multicriteria optimization problems. One approach is to introduce a single criterion that somehow incorporates the many criteria of the problem (see, e.g., Wood [19]). Another technique uses the notion of Pareto optimality to achieve a balance between the objectives (see, for example, Sawaragi, Nakayama, and Tanino [12]). The approach of goal programming selects one objective to serve as an optimization objective and turns the other objectives into constraints by setting bounds or "goals" for them. Finally, a subset of multilevel problems, known as lexicographic optimization problems, involves the notion of lexicographic comparison; see, for instance, Ben-Israel, Ben-Tal, and Zlobec [5].

We consider yet another approach, namely, to restate the multiobjective problem as a multilevel optimization problem. This approach has not been extensively used because, to the authors' knowledge, efficient algorithms for general nonlinear multilevel optimization have not yet been discovered. In addition, due to the theoretical complexity of the problem, a theoretical basis for the general problem has not been developed as yet. The general problem of MLO follows.

Let $f_{m}, \ldots, f_{1}$ be the problem objectives, arranged in the order of increasing significance, i.e., $f_{1}$ is the most important objective, while $f_{m}$ is the least important objective. Note that this significance does not need to be quantified in any way other than the establishment of the order. Then the formulation is:

Problem MLO:

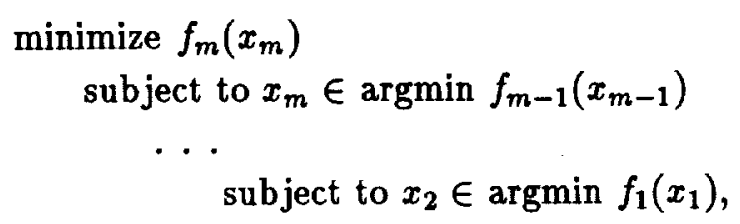

where "argmin" denotes the set of minima of a particular $f_{i}$, and $f_{1}, \ldots, f_{m}: \Re^{n} \rightarrow \Re$ are sufficiently smooth. The formulation can be easily extended to include constraints, but in the scope of this discussion we shall address unconstrained objectives.

Thus the MLO formulation gives us a way to "regularize" the ill-defined problem of multicriteria optimization. Here the engineering insight will enter into defining the order in which the optimization levels are stated. The reader will see that if the objective values of all the objectives except the most important one were known at a solution to an MLO problem, then one would have a goal 
program. Thus, it is possible to view MLO as related to goal programming except that the order of importance of the objectives needs to be specified rather than goals for each objective value.

Existing work in multilevel and, in particular, bilevel optimization (see Vicente and Calamai [18] for a review) deals only with functions under extremely strong assumptions of convexity and has many theoretical difficulties. We are approaching this problem with cautious optimism because in recent work (see Alexandrov [1], Alexandrov and Dennis [2], [3]), algorithms for multilevel optimization of problems with special structure have been shown to exhibit global convergence under reasonable assumptions.

In this paper we will use the general bilevel optimization problem to discuss issues in MLO. First, we remark in passing that for the two objectives, there are two different problems determined by the assignment of the order to the criteria:

$$
\begin{array}{ll}
\text { Problem 1: } & \text { minimize } f_{1}(x) \\
& \text { subject to } x \in \operatorname{argmin}\left\{f_{2}(y)\right\},
\end{array}
$$

and

$$
\begin{array}{ll}
\text { Problem 2: } & \text { minimize } f_{2}(x) \\
& \text { subject to } x \in \operatorname{argmin}\left\{f_{1}(y)\right\} .
\end{array}
$$

The two problems will almost certainly have different answers. In fact, there are simple examples of one problem being well-posed, while the other is ill-posed. We contend that engineering judgement and insight into the problem is likely to produce a correct or optimal order. In the contrary case, establishing the right order is likely to lead to engineering insight.

We are proposing two formal algorithms for the bilevel and multilevel optimization problems. One algorithm is an extension of the multilevel algorithms in Alexandrov [1] and it arrives from the current approximation of the solution to the next approximation by computing a sequence of solutions to the minimization subproblems restricted to smaller and smaller dimensional subspaces. The second algorithm arrives at the next estimate of the solution by solving a sequence of local optimization subproblems each of which will serve to set a local "goal" in defining the region of sufficient decrease in the merit function for the final local optimization subproblem. We have a new, promising, merit function that will allow us to evaluate the progress of the algorithm toward a solution.

\section{Nonlinear Programming Preliminaries}

In this section we define a number of concepts from unconstrained optimization, that enter both into practical conditions imposed on the steps in an optimization algorithm and into algorithm convergence analysis. We also briefly describe the multilevel algorithms for nonlinear optimization on which the algorithms proposed here are based. Consider the following unconstrained minimization problem.

Problem UNC:

minimize $f(x)$

subject to $x \in \Re^{n}$, 
where $f: \Re^{n} \rightarrow \Re$ is continuously differentiable.

Newton's method and its variations form a standard class of local solution methods for UNC, and they can be stated as follows:

1. Initialize;

2. Do until convergence:

Build a local model:

$$
\phi_{c}(s)=f\left(x_{c}\right)+\nabla f\left(x_{c}\right)^{T} s+\frac{1}{2} s^{T} H_{c} s
$$

Minimize $\phi_{c}(s)$ to obtain $s_{c}$;

Set $x_{+}=x_{c}+s_{c}$;

3. End.

Here $x_{c}$ and $x_{+}$denote the current and the next approximation to a solution, respectively, and $H_{c}$ is an approximation to the Hessian of $f$ at $x_{c}$, but not necessarily the true Hessian.

Trust region algorithms form one of the major approaches designed to improve the global behavior of such local model based algorithms. At each iteration, a typical trust-region algorithm for solving problem UNC finds a trial step by solving the following trust-region subproblem approximately:

$$
\begin{aligned}
& \text { minimize } f\left(x_{c}\right)+\nabla f\left(x_{c}\right)^{T} s+\frac{1}{2} s^{T} H_{c} s \\
& \text { subject to }\|s\| \leq \delta_{c},
\end{aligned}
$$

where $\delta_{c}>0$ is the trust-region radius, and $\|\cdot\|$ denotes the $\ell_{2}$ norm. The idea is to model the objective function in a restricted region and to accept the trial step when the quadratic model adequately predicts the behavior of the function, and to recompute the step in a smaller region if it does not.

Detailed treatment of the trust-region approach to unconstrained optimization and nonlinear equations can be found in Dennis and Schnabel [6], Sorensen [17], Moré [8], Moré and Sorensen [9], Powell [7], and Shultz, Schnabel and Byrd[13].

Trust-region algorithms have been successfully extended to solve the general nonlinear constrained optimization problem. In particular, the local step in the successive quadratic programming (SQP) method is found by computing a minimizer of the quadratic model of the Lagrangian at the current point, subject to linearized constraints. A trust-region algorithm based on SQP adds the trust-region constraint to the subproblem and additional constraints designed to ensure that the trust-region constraint and the linearized constraints are consistent. We shall see that the algorithms proposed here may be viewed as a generalization of the SQP approach to bilevel and multilevel optimization.

\subsection{Merit Functions}

In order to evaluate a trial step, trust-region algorithms use merit functions, which are functions related to the problem in such a way that the improvement in the merit function signifies progress toward the solution of the problem.

For unconstrained minimization, a natural choice for a merit function is the objective function itself. Let

$$
\phi_{c}(s)=f\left(x_{c}\right)+\nabla f\left(x_{c}\right)^{T} s+\frac{1}{2} s^{T} H_{c} s
$$


denote the quadratic model of the merit function. We define two related functions.

The actual reduction is defined as

$$
\operatorname{ared}_{c}\left(s_{c}\right)=f\left(x_{c}\right)-f\left(x_{c}+s_{c}\right)
$$

and the predicted reduction is defined as

$$
\begin{aligned}
\operatorname{pred}_{c}\left(s_{c}\right) & =\phi_{c}(0)-\phi_{c}\left(s_{c}\right) \\
& =-\nabla f\left(x_{c}\right)^{T}\left(s_{c}\right)-\frac{1}{2} s_{c}^{T} H_{c} s_{c}
\end{aligned}
$$

so that the predicted reduction in the merit function is an approximation to the actual reduction in the merit function.

The standard way to evaluate the trial step in trust-region methods is to consider the ratio of the actual reduction to the predicted reduction. A value lower than a small predetermined value causes the step to be rejected. Otherwise the step is accepted.

For nonlinear systems of equations, the norm of the residuals serves as a merit function. For the constrained optimization, the merit function is some expression that involves both the objective function and the constraints.

\subsection{Fraction of Optimal Decrease and Fraction of Cauchy Decrease}

To assure global convergence of a trust-region algorithm for problem UNC, the trial step is required to satisfy a fraction of Cauchy decrease (FCD) condition. This mild condition means that the trial step, $s_{c}$, must predict at least a fraction of the decrease predicted by the Cauchy step, which is the steepest descent step for the model within the trust region. We must have for some fixed $\kappa_{1}>0$

$$
\operatorname{pred}\left(s_{c}\right)=\phi_{c}\left(s_{c}\right)-\phi_{c}(0) \leq \kappa_{1}\left[\phi_{c}\left(s_{c}^{C P}\right)-\phi_{c}(0)\right]
$$

where

$$
\begin{gathered}
s_{c}^{C P}=-\alpha_{c}^{C P} \nabla f\left(x_{c}\right) \text { with } \\
\alpha_{c}^{C P}= \begin{cases}\frac{\left\|\nabla f\left(x_{c}\right)\right\|^{2}}{\nabla f\left(x_{c}\right)^{T} H_{c} \nabla f\left(x_{c}\right)} & \text { if } \frac{\left\|\nabla f\left(x_{c}\right)\right\|^{3}}{\nabla f\left(x_{c}\right)^{T} H_{c} \nabla f\left(x_{c}\right)} \leq \delta_{c} \\
\left\|\nabla f\left(x_{c}\right)\right\| & \text { otherwise. }\end{cases}
\end{gathered}
$$

See Dennis and Schnabel [6], pp. 139-141, for details on the Cauchy point.

A stronger condition, the fraction of optimal decrease property (FOD), allows one to prove stronger convergence results. A step $s_{c}$ is said to satisfy FOD if it predicts at least a fraction of the decrease predicted by the optimal solution of the trust-region subproblem, i.e., for some fixed $\kappa_{2}>0$ we have

$$
\operatorname{pred}\left(s_{c}\right)=\phi_{c}\left(s_{c}\right)-\phi_{c}(0) \leq \kappa_{2}\left[\phi_{c}\left(s_{c}^{O P T}\right)-\phi_{c}(0)\right],
$$

where $s_{c}^{O P T}$ solves the trust-region subproblem exactly.

The FCD condition is satisfied by all variants of the dogleg method and by restricted subspace methods, for example. The stronger FOD condition is satisfied by most algorithms that attempt to accurately minimize the local model on the trust region, for instance, by Levenberg-Marquardt type methods. 


\subsection{Convergence Results}

Powell's global convergence theorem (see Powell [7]) for any unconstrained minimization trustregion algorithm shows the power of trust-region globalization ideas. It states that if $f$ is uniformly continuously differentiable and $\left\{H_{i}\right\}$ are only assumed to be uniformly bounded, then the sequence of iterates generated by a FCD trust-region algorithm is well-defined and satisfies

$$
\liminf _{i \rightarrow \infty}\left\|\nabla f\left(x_{i}\right)\right\|=0 .
$$

Sorensen [17] has shown stronger convergence results for trust-region algorithms with steps that satisfy FOD. Specifically, he has shown that if the Hessian is Lipschitz continuous, and if exact Hessians are used in the local models, then any limit point of the iterates satisfies second order necessary conditions, i.e., has a positive semidefinite Hessian. Furthermore, under some reasonable additional assumptions, the iteration sequence converges q-quadratically to a second order necessary point for UNC.

Detailed treatment of the unconstrained minimization theory and practice can be found in Moré [8], Moré and Sorensen [9], Sorensen [17], and Shultz, Schnabel and Byrd [13].

\subsection{Multilevel Methods for Nonlinear Equations Equality Constrained Opti- mization}

The algorithms introduced here are based on the recently proposed class of multilevel algorithms for equality constrained optimization and nonlinear equations (see Alexandrov [1], Alexandrov and Dennis [2], [3]).

The algorithms of that class use trust regions as a globalization startegy, they have been shown to be globally convergent under reasonable assumptions. They have the following characteristics:

- The constraints of the problem can be partitioned into blocks by the user in any manner suitable to an application, or in any arbitrary manner at all.

- The analysis of the methods assumes certain standard smoothness and boundedness properties, but no other assumptions are made on the structure of the problem.

- The algorithms solve at each iteration progressively smaller dimensional subproblems to arrive at the trial step.

- The trial steps computed by the algorithm are required to satisfy very mild conditions, both theoretically and computationally. In fact, the substeps comprising the trial step can be computed in the subproblems using different optimization algorithms. The substeps are only required to satisfy a mild decrease condition for the subproblems and a reasonable boundedness condition-both satisfied in practice by most methods of interest.

The proposed multilevel class of algorithms differs from the conventional algorithms in that its major iteration involves computing an approximate solution of not one model over a single restricted region, but of a sweep of models, each approximately minimized over its own restricted region. Each model approximates a block of constraints and, finally, the objective function, restricted to certain subspaces. The algorithms proposed in this work follow this principle with equality constraints replaced by one or more levels of optimization problems. 


\section{Formulations and Algorithms}

In this section we consider some formulations of the bilevel problem

Problem BLO:

minimize $f_{2}(x)$

subject to $x \in \operatorname{argmin}\left\{f_{1}(x)\right\}$

and discuss their properties, including necessary conditions for minima. Then we suggest algorithms suitable for the specific formulations.

In our discussions we assume no convexity, unless specified otherwise. We assume that all functions are at least twice continuously differentiable and that $f_{1}$ is bounded from below.

The formulation, which we call BLO, means that among the minima of $f_{1}$ we wish to find a point, for which the value of $f_{2}$ is the lowest. There are three cases.

1. $f_{1}$ is strictly convex. There is one global minimizer of $f_{1}$, and, therefore, the feasible point is the solution of the problem.

2. The set of minima of $f_{1}$ is a set of disjoint points. Since algorithms for continuous nonlinear optimization are guaranteed, in general, to find only local solution, this case, in effect, is identical to the first one.

3. The set of minima of $f_{1}$ has a nonempty relative interior.

Since the first two cases are degenerate as bilevel problems, we shall consider only the third one from now on.

Suppose the point $x_{*} \in \Re^{n}$ solves the innermost problem of problem BLO, i.e., $x_{*}$ is an unconstrained minimizer of $f_{1}(x)$. Let $f_{1}^{*}$ be the corresponding value of $f_{1}$. Then our problem BLO would seem equivalent to the following problem:

$$
\begin{aligned}
& \operatorname{minimize} f_{2}(x) \\
& \text { subject to } f_{1}(x)=f_{1}^{*} .
\end{aligned}
$$

However, this formulation will not have a Lagrange multiplier at the minimum because $\nabla f_{1}\left(x_{*}\right)=$ 0 and thus the first order necessary conditions will hold only if $\nabla f_{2}\left(x_{*}\right)=0$ coincidentally. Therefore, the problem is ill-posed in this form.

\subsection{Approach Based on First Order Necessary Conditions for a Solution}

Now consider the following formulation based on the first order necessary condition for the innermost problem:

\section{Problem FOC:}

minimize $f_{2}(x)$

subject to $\nabla f_{1}(x)=0$.

Clearly, for a convex $f_{1}$ the formulations BLO and FOC are equivalent. To study the relation between the formulation in the general case, we introduce the notion of constraint qualifications. 
In order to determine optimality in constrained optimization, it is necessary to study the behavior of the objective function along feasible perturbations. Conditions that allow us to characterize feasible perturbations completely are known as constraint qualifications. Constraint qualifications may take different forms, some of them purely theoretical. A common practical constraint qualification in nonlinear programming is regularity, which is the assumption of full rank for the Jacobian of the constraint system. As we mentioned, regularity fails for the most obvious reformulation of problem BLO.

For problem FOC, the Jacobian of the constraint system is $\nabla^{2} f_{1}(x)$. It is a square matrix, positive semidefinite at a solution of problem BLO. We assume that the matrix is singular, for otherwise the inner problem would have an isolated minimum, resulting in the degenerate case. We claim that a reasonable constraint qualification for problems FOC and BLO is to require $\nabla^{2} f_{1}(x)$ to have constant rank in a neighborhood of the solution. This assumption is a natural extension of the full-rank assumption for rectangular matrices and is based on the results in continuity of generalized inverses (see Campbell and Meyer [11], for example).

Let $x_{*}$ solve the bilevel optimization problem BLO. Assuming the constant rank constraint qualification, it can be shown that the first order necessary conditions for an optimum of problem BLO and problem FOC is:

$$
\begin{aligned}
& \nabla f_{2}\left(x_{*}\right)+\lambda^{T} \nabla^{2} f_{1}\left(x_{*}\right)=0 \\
& \nabla f_{1}\left(x_{*}\right)=0 \\
& \nabla^{2} f_{1}\left(x_{*}\right) \text { is positive semidefinite. }
\end{aligned}
$$

We believe that adding the condition of positive semidefiniteness of $\nabla^{2} f_{2}\left(x_{*}\right)$ on the null space of $\nabla^{2} f_{1}\left(x_{*}\right)$ to the above conditions together with our constraint qualification will constitute the second order necessary optimality conditions for problem BLO.

We also believe that for general nonlinear $f_{1}$ and $f_{2}$, if $x_{*}$ solves problem FOC and it is feasible for problem BLO, then it also solves problem BLO.

Thus, it is reasonable to attack problem BLO by solving problem FOC if we ensure that the solution is a minimum of $f_{1}$ and therefore feasible for BLO. In practice, we propose to solve problem FOC by the multilevel algorithm for equality constrained optimization introduced in Alexandrov [1] (see also Alexandrov and Dennis [2], [3]). To measure progress toward a solution and to ensure that it is feasible with respect to problem BLO, we propose to attempt two merit functions:

$$
P_{1}(x ; \rho)=f_{2}(x)+\rho f_{1}(x)
$$

and

$$
P_{2}(x ; \rho)=f_{2}(x)+\lambda^{T} \nabla f_{1}(x)+\rho f_{1}(x)^{2} .
$$

The first merit function is an analog of the objective function used as a merit function in unconstrained minimization. The second one is an analog of the augmented Lagrangian used as a merit function in constrained optimization.

A possible drawback of this approach is that second order information may be necessary for the for the algorithm. On the positive side, the analysis of the multilevel algorithms for constrained optimization [1] will apply to the approach after minor modifications. Both in theory and in practice, the steps would have to satisfy the mild FCD condition for the subproblem that they solve. In addition, this formulation is easy to extend to the general multilevel optimization problem. 


\subsection{Approach Based on Successive Decrease Conditions}

Now that we have the first order necessary conditions for a solution of problem BLO, let us consider an approach that will require no explicit reformulation of the problem.

Sorensen [17] has shown that if we use the exact Hessians and steps that satisfy the FOD condition in a trust-region algorithm for unconstrained minimization, then the algorithm converges to a point that satisfies the first order necessary conditions for a minimum. Thus, it is reasonable to expect - though it must be verified - that if we apply an FOD method to our problem, we should have convergence to a point satisfying first order necessary conditions.

The algorithm we propose for bilevel optimization can be stated as follows:

Compute the trial step for problem BLO to produce an FOD on the quadratic model of $f_{2}$ subject to producing FOD on the quadratic model of $f_{1}$.

A version that imposes a milder FCD type condition on the step is also of interest.

In practice, the algorithm would be implemented in the following way. The inner problem would be solved by a conventional trust-region approach to unconstrained minimization to produce the FOD "goal" for the quadratic model of $f_{1}$ about the current point. Then the outer problem would be solved in the null space of $\nabla^{2} f_{1}\left(x_{c}\right)$ subject to the condition that the step produce the FOD condition in the model of $f_{2}$.

This approach can be extended to any number of levels in a natural way. Clearly, if the objective values of all the objectives except the most important one were known at a solution to an MLO problem, then one would have a goal program. One can think of our algorithm as a way to set goals adaptively for each iteration.

We propose to use the same two merit functions as in the previous subsection.

\section{Concluding Remarks}

We proposed two approaches to solving the bilevel optimization problem, which can be easily extended to general multilevel problem with an arbitrary number of levels and with constraints.

The main difficulties of the multilevel formulations have always been the possible intractability of the feasible set for the problem and in showing the existence of search directions under reasonable assumptions. We proposed a constraint qualification which is a reasonable extension of the standard constraint qualification for constrained nonlinear optimization. This qualification has allowed us to establish first order necessary conditions for a solution of the bilevel problem. These conditions give us hope that the algorithms will be of practical use. Our next step is thorough practical testing of the algorithms combined with further theoretical investigations.

\section{References}

[1] Natalia Alexandrov. Multilevel Algorithms for Nonlinear Equations and Equality Constrained Optimization. PhD thesis, Rice University, Department of Computational and Applied Mathematics, May 1993. 
[2] Natalia Alexandrov and J. E. Dennis, Jr. A class of general trust-region multilevel algorithms for systems of nonlinear equations: Global convergence theory. To be submitted to SIAM Journal of Optimization.

[3] Natalia Alexandrov and J. E Dennis, Jr. Multilevel algorithms for nonlinear optimization. In Proceedings of the Workshop on Optimal Design and Control, Blacksburg, VA, April 8-9 1994. To appear. Also available as ICASE Technical Report94-53 (ICASE, Mail Stop 132C, NASA Langley Research Center, Hampton, VA 23681-0001) and as Technical Report TR94-24 (Department of Computational and Applied Mathematics, Rice University, P.O. Box 1892, Houston, Texas 77251-1892).

[4] J-F M. Barthelemy. Engineering applications of heuristic multilevel optimization methods. Technical report, NASA Langley Research Center, Hampton, VA 23665, October 1988. NASA TM-101504.

[5] A. Ben-Israel, A. Ben-Tal, and S. Zlobec. Optimality in Nonliear Programming: a Feasible Directions Approach. John Wiley and Sons, New York, 1981.

[6] J. E. Dennis, Jr. and Robert B. Schnabel. Numerical Methods for Unconstrained Optimization and Nonlinear Equations. Prentice-Hall, Englewood Cliffs, New Jersey, 1983.

[7] M. J. D. Powell. Convergence properties of a class of minimization algorithms. In O. L. Mangasarian, R. R. Meyer, and S. M. Robinson, editors, Nonlinear Programming 2. Academic Press, 1975.

[8] J. J. Moré. Recent developments in algorithms and software for trust region methods. In A. Bachem, M. Grötschel, and B. Korte, editors, Mathematical Programming: The State of the Art. Springer-Verlag, 1983.

[9] J. J. Moré and D. C. Sorensen. Computing a trust region step. SIAM J. Sci. Stat. Comput., 4(3):553-572, September 1983.

[10] S. L. Padula and K. C. Young. Simulator for multilevel optimization research. Technical report, NASA Langley Research Center, Hampton, VA 23665, June 1986. NASA TM-87751.

[11] S. L. Campbell and C. P. Meyer, Jr. Generalized Inverses of Linear Transformations. Dover Publications, 1991.

[12] Y. Sawaragi, H. Nakayama, and T. Tanino. Theory of Multiobjective Optimization. Academic Press, New York, 1985.

[13] Gerald A. Shultz, Robert B. Schnabel, and Richard H. Byrd. A family of trust-region-based algorithms for unconstrained minimization with strong global convergence properties. SIAM J. Numerical Analysis, 22(1):47-67, February 1985.

[14] Jaroslaw Sobieszczanski-Sobieski. Aircraft optimization by a system approach: Achievments and trends. Technical report, NASA Langley Research Center, Hampton, VA 23665, May 1992. NASA TM-107622. 
[15] Jaroslaw Sobieszczanski-Sobieski, Benjamin James, and Augustine Dovi. Structural optimization by multilevel decomposition. In Proceedings of the AIAA/ASME/ASCE/AHS 24th Structures, Structural Dynamics and Materials Conference, Lake Tahoe, Nevada, May 2-4 1983. AIAA Paper No. 83-0832-CP.

[16] Jaroslaw Sobieszczanski-Sobieski, Benjamin B. James, and Michael F. Riley. Structural sizing by generalized, multilevel optimization. AIAA Journal, 25(1), January 1987.

[17] D. C. Sorensen. Newton's method with a model trust region modification. SIAM J. Numerical Analysis, 19(2):409-426, April 1982.

[18] Luis N. Vicente and Paul H. Calamai. Bilievel and multilevel programming: A bibliography review. Technical Report 197-0-180393, Department of Systems Design Engineering, University of Waterloo, 1988.

[19] D. J. Wood. An Interactive Approach for Solving Multi-Objective Optimization Problems. PhD thesis, Rice University, 1985. also available as TR85-05, Department of Computational and Applied Mathematics, Rice University, P.O. Box 1892, Houston, Texas 77251-1892. 




\begin{tabular}{|c|c|c|c|}
\hline \multicolumn{3}{|c|}{ REPORT DOCUMENTATION PAGE } & $\begin{array}{l}\text { Form Approved } \\
\text { OMB No. } 0704-0188\end{array}$ \\
\hline \multicolumn{4}{|c|}{ 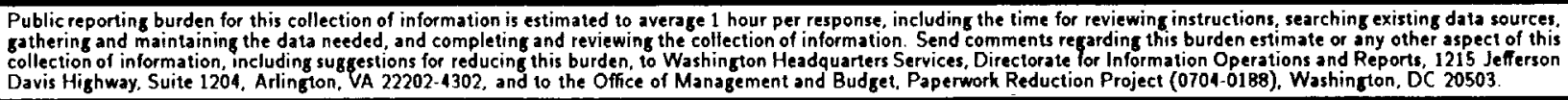 } \\
\hline 1. AGENCY USE ONLY(Leave blank) & $\begin{array}{l}\text { 2. REPORT DATE } \\
\text { September } 1994\end{array}$ & \multicolumn{2}{|c|}{$\begin{array}{l}\text { 3. REPORT TYPE AND DATES COVERED } \\
\text { Contractor Report }\end{array}$} \\
\hline \multicolumn{2}{|c|}{$\begin{array}{l}\text { 4. TITLE AND SUBTITLE } \\
\text { ALGORITHMS FOR BILEVEL OPTIMIZATION }\end{array}$} & & \multirow[t]{2}{*}{$\begin{array}{l}\text { 5. FUNDING NUMBERS } \\
\text { C NAS1-19480 } \\
\text { WU } 505-90-52-01\end{array}$} \\
\hline \multicolumn{3}{|l|}{$\begin{array}{l}\text { 6. AUTHOR(S) } \\
\text { Natalia Alexandrov } \\
\text { J. E. Dennis, Jr. }\end{array}$} & \\
\hline \multicolumn{2}{|c|}{$\begin{array}{l}\text { 7. PERFORMING ORGANIZATION NAME(S) AND ADORESS(ES) } \\
\text { Institute for Computer Applications in Science } \\
\text { and Engineering } \\
\text { Mail Stop 132C, NASA Langley Research Center } \\
\text { Hampton, VA 23681-0001 }\end{array}$} & & $\begin{array}{l}\text { 8. PERFORMING ORGANIZATION } \\
\text { REPORT NUMBER } \\
\text { ICASE Report No. } 94-77\end{array}$ \\
\hline \multicolumn{2}{|c|}{$\begin{array}{l}\text { 9. SPONSORING/MONITORING AGENCY NAME(S) AND ADDRESS(E } \\
\text { National Aeronautics and Space Administration } \\
\text { Langley Research Center } \\
\text { Hampton, VA } 23681-0001\end{array}$} & $\begin{array}{l}\text { 10. SPONS } \\
\text { AGENC } \\
\text { NASA } \\
\text { ICASE }\end{array}$ & $\begin{array}{l}\text { 10. SPONSORING/MONITORING } \\
\text { AGENCY REPORT NUMBER } \\
\text { NASA CR-194980 } \\
\text { ICASE Report No. } 94-77\end{array}$ \\
\hline \multicolumn{4}{|c|}{$\begin{array}{l}\text { 11. SUPPLEMENTARY NOTES } \\
\text { Langley Technical Monitor: Michael F. Card } \\
\text { Final Report } \\
\text { To appear in Proc. of 5th AIAA/NASA/USAF/ISSMO Symp. on Multidiscipl }\end{array}$} \\
\hline \multicolumn{2}{|c|}{$\begin{array}{l}\text { 12a. DISTRIBUTION/AVAILABILITY STATEMENT } \\
\text { Unclassified-Unlimited } \\
\text { Subject Category } 64\end{array}$} & & 12b. DISTRIBUTION CODE \\
\hline \multicolumn{4}{|c|}{$\begin{array}{l}\text { 13. ABSTRACT (Maximum } 200 \text { words) } \\
\text { General multilevel nonlinear optimization problems arise in design of complex systems and can be used as a means of } \\
\text { regularization for multicriteria optimization problems. Here for clarity in displaying our ideas, we restrict ourselves } \\
\text { to general bilevel optimization problems, and we present two solution approaches. Both approaches use a trust- } \\
\text { region globalization strategy, and they can be easily extended to handle the general multilevel problem. We make } \\
\text { no convexity assumptions, but we do assume that the problem has a nondegenerate feasible set. We consider } \\
\text { necessary optimality conditions for the bilevel problem formulations and discuss results that can be extended to } \\
\text { obtain multilevel optimization formulations with constraints at each level. }\end{array}$} \\
\hline \multirow{2}{*}{\multicolumn{3}{|c|}{$\begin{array}{l}\text { 14. SUBJECT TERMS } \\
\text { Multilevel Algorithms, Multilevel Optimization, Bilevel Optimization, Lexicographic } \\
\text { Optimization, Multicriteria Optimization, Constrained Optimization, Global Conver- } \\
\text { gence, Globalization, Trust Region, Nonlinear Programming, Constraints }\end{array}$}} & $\begin{array}{c}\text { 15. NUMBER OF PAGES } \\
12\end{array}$ \\
\hline & & & $\begin{array}{c}\text { 16. PRICE CODE } \\
\mathrm{A03}\end{array}$ \\
\hline $\begin{array}{l}\text { 17. SECURITY CLASSIFICATION } \\
\text { OF REPORT } \\
\text { Unclassified }\end{array}$ & $\begin{array}{l}\text { 18. SECURITY CLASSIFICATION } \\
\text { OF THIS PAGE } \\
\text { Unclassified }\end{array}$ & $\begin{array}{l}\text { 19. SECURITY CLASSIFICATION } \\
\text { OF ABSTRACT }\end{array}$ & $\begin{array}{l}\text { 20. LIMITATION } \\
\text { OF ABSTRACT }\end{array}$ \\
\hline NSN 7540-01-280-5500 & & & $\begin{array}{l}\text { Standard Form 298(Rev. 2-89) } \\
\text { Prescribed by ANSI Std. Z39-18 } \\
298-102\end{array}$ \\
\hline
\end{tabular}


National Aeronautics and Space Administration

Langley Research Center

Mail Code 180

Hampton, VA 23681-00001

BULK RATE

POSTAGE \& FEES PAID

NASA

Ometal Buelnates

Penalyy for Priveno Uea, 8300

Permit No. G-27 
